\title{
JOINT DOMAIN LOCALIZED PROCESSING USING MEASURED SPATIAL STEERING VECTORS ${ }^{*}$
}

\author{
Raviraj S. Adve \\ Research Associates for Defense Conversion, Inc. \\ 10002 Hillside Terrace, Marcy, NY 13403
}

\author{
Michael C. Wicks \\ Air Force Research Laboratory, Sensors Directorate \\ 26 Electronic Parkway, Rome, NY 13441-4514
}

\begin{abstract}
The Joint Domain Localized (JDL) adaptive processing algorithm mitigates interference in a localized processing region in the angle-Doppler domain. Spatial and temporal steering vectors transform the data collected in the space-time domain to the angle-Doppler domain. However, the JDL algorithm depends on the assumption that the spatial and temporal steering vectors form an orthogonal set. In practice, due to the mutual coupling between antenna elements, the spatial steering vectors do not form an orthogonal set. This paper presents a modification of the JDL algorithm to account for the non-orthogonality of these steering vectors. Examples from the MCARM database demonstrate the improvement in detection capability.
\end{abstract}

\subsection{INTRODUCTION}

The detection of small, slow moving targets by airborne surveillance radar requires the suppression of strong, dynamic and possibly inhomogeneous interference in real time. Space-time adaptive processing (STAP) techniques promise to be the best means to suppress such interference. Consider a phased array antenna with $N$ spatial channels, possibly subarrays of a larger array, with $M$ pulses per coherent processing interval (CPI). The most straight forward, and optimal, STAP algorithm uses the (known) $N M$ dimensional covariance matrix of the interference to obtain $N M$ complex weights that minimize the expected squared error with respect to the desired signal [Brennan, 1973]. In a practical situation, this covariance matrix is unknown and is estimated by averaging over secondary data from range cells surrounding the range cell of interest. For an accurate estimate, about $2 N M$ to $3 N M$ independent, identically distributed (i.i.d.) data samples are required. The problem with this joint domain approach is that in practice, it is difficult to obtain such a large number of i.i.d. samples. Further, the computation expense rises as the third power of the number of weights. Hence, even if i.i.d samples are available, the computation load makes this optimal joint domain algorithm impractical.

To overcome the drawbacks of the joint domain algorithm, researchers have tried to limit the number of adaptive weights so as to reduce problems associated with sample support and computation expense. Wang and Cai [Wang, 1994] introduced the Joint Domain Localized (JDL) algorithm, a post-Doppler, beamspace, approach that adaptively processes the radar data in the angle-Doppler domain. An inner product with the spatial and temporal steering vectors transforms space-time data to the angle-Doppler domain. The spatial steering vector associated with a particular angle is the complex conjugate of the normalized vector of measured voltages due to a far field point source at that angle. Similarly, the temporal steering vector associated with a Doppler frequency is computed using the vector of vcltages due a calibrated source offset from the carrier by that Doppler frequency. This transformation localizes the signal and interference in angle-Doppler space. As such, adaptive processing may be performed in a localized processing region centered about the test angle-Doppler bin. Hence, interference suppression is achieved using a limited number of adaptive weights, in turn, requiring limited secondary data.

In developing the JDL algorithm, the authors assume the receiving antenna to be a uniformly spaced, linear array of ideal, isotropic, point elements. Under this assumption, the inverse Fourier coefficients form the set of spatial and temporal steering vectors. This allows for the use of Discrete Fourier Transforms (DFT) to transform

\footnotetext{
* Presented at the IEEE 1998 National Radar Conference, Dallas, TX, 12-13 May 1998. This work was sponsored by the Air Force Research Laboratory, Sensors Directorate, under contract F30602-97-C-0006.
} 
from space domain to the angle domain and the time domain to the Doppler domain. The orthogonality of the Fourier coefficients localizes the target space-time signal to a single point in the angle-Doppler domain.

In practice, however, the elements of the array are not isotropic point sensors. Due to their physical size, the elements not only spatially sample incident fields, but also re-radiate them. The re-radiated fields interact with the other elements manifested as mutual coupling effects. Further, the assumption of a linear array is restrictive. The array may be planar, allowing for degrees of freedom in azimuth and elevation. In practice, the Fourier coefficients do not form the spatial steering vector and a DFT does not transform the space domain to the angle domain. The spatial steering vectors have to be either measured or obtained using numerical electromagnetic analysis. It is these measured steering vectors that transform the space domain to the angle domain. Unlike in the ideal case, the measured steering vectors do not form an orthogonal set. Mutual coupling does not affect the temporal steering vectors.

This paper modifies the traditional JDL algorithm of [Wang, 1994] to account for the non-orthogonality of measured steering vectors. Examples using measured data from the Multi-Channel Airborne Radar Measurements (MCARM) database [Sloper, 1996] illustrate the improvement in detection performance. The database contains clutter and signal measurements collected via an airborne radar over many flights with multiple acquisitions on each flight. The radar antenna is a $22(2 \times 11)$ rectangular array. Also provided with the database is a set of measured spatial steering vectors. Wang [Wang, 1995] and Melvin and Himed [Melvin, 1996] applied the JDL algorithm to the MCARM data and use the measured steering vectors to transform the space domain to the angle domain. However, they ignore the fact that the spatial steering vectors are not orthogonal. By comparing the performance of the JDL algorithm before [Wang, 1995, Melvin, 1996] and after the proposed modifications, it is shown that the nonorthogonality of the steering vectors plays a significant role in practical antenna arrays.

Section 2.0 introduces the JDL algorithm as applied to the ideal case of a linear array of isotropic, point sensors and details the use of non-orthogonal steering vectors. Section 3.0 presents examples to illustrate the improvement in processing performance obtained by the modifications proposed in this paper. Finally, section 4.0 presents some conclusions and a summary of the results presented here.

\subsection{THE JDL ALGORITHM}

Consider an array of $N$ antenna elements receiving an incident plane wave. Each receiver collects $M$ data samples corresponding to $M$ pulses in the CPI. For each range bin, the data is a vector $\mathbf{X}$ of length $M N$, containing entries numbered $(m-1) N+1$ to $m N$, corresponding to the returns from the $m$ th pulse. In range cells without a target, $\mathbf{X}=\mathbf{C}+\mathbf{N}$ and in range cells with a target, $\mathbf{X}=\alpha \mathbf{S}+\mathbf{C}+\mathbf{N}$, where, $\alpha$ is the target amplitude, $\mathbf{S}$ is the space-time steering vector, $\mathbf{C}$ the vector of interference sources and $\mathbf{N}$ the noise vector. Each term in the vector $\alpha \mathbf{S}$, corresponding to a target at angle $\theta$ and radial velocity component $v$, is given by

$$
S_{m(N+1)+n}=\alpha e^{\left[j 2 \pi(n-1) f_{1}+j 2 \pi(n-1) f_{s}\right]}
$$

where, $f_{t}$ is the normalized Doppler frequency and $f_{s}$ the normalized spatial frequency given by $f_{s}=d \sin \theta / \lambda$, $f_{t}=2 v / \lambda=f_{\mathrm{d}} / P R F$, where $P R F$ is the pulse repetition frequency, $f_{d}$ the Doppler frequency, $\lambda$ the design wavelength of the array and $d$ the spacing between elements.

The contribution of the target can be written in terms of the spatial steering vector $\left(S_{s}\right)$ and temporal steering vector $\left(S_{t}\right)$, i.e. $\mathbf{S}=\alpha S_{s} \otimes S_{t}$ where $\otimes$ denotes the Kronecker product. In the case of a linear array of ideal, isotropic elements [Ward, 1994],

$$
\begin{aligned}
& S_{s}=\left[1 e^{j 2 \pi f_{s}} e^{j 2 * 2 \pi f_{s}} e^{j 3 * 2 \pi f_{s}} \ldots \ldots e^{j(N-1) * 2 \pi f_{s}}\right]
\end{aligned}
$$

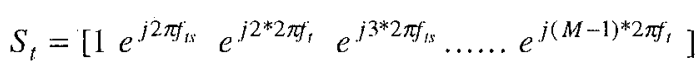




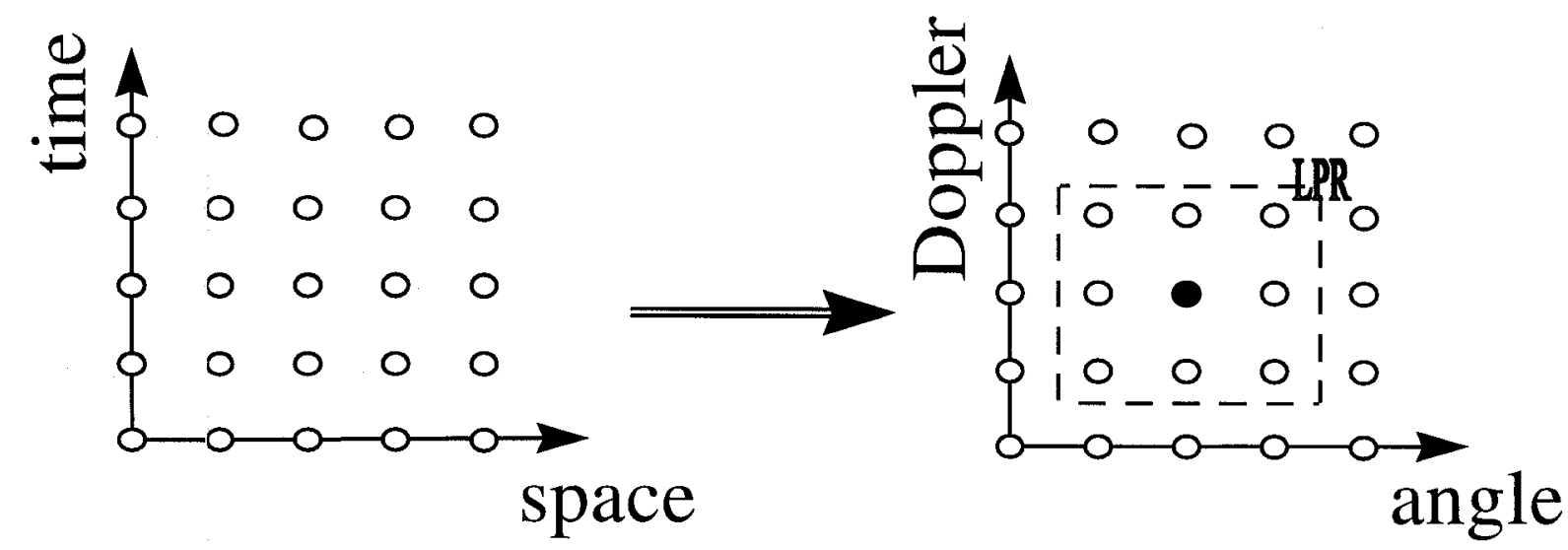

Figure 1: Transformation of the space-time domain to the angle-Doppler domain

Equations (2) and (3) indicate that the components of the spatial and temporal steering vectors are the Fourier coefficients. Therefore, an inner product with the space-time steering vector is equivalent to a two dimensional DFT. The 2-D DFT transforms the space-time data $\mathbf{X}$ to the angle-Doppler data $\chi$. Since the rows of a DFT matrix are orthogonal, the 2-D DFT transforms multi-channel, multi-pulse target data to a single point in the angle-Doppler domain, i.e., the signal is localized. This localization of the signal and interference implies that interference suppression can be achieved using a limited number of adaptive weights.

The JDL algorithm forms a localized processing region (LPR), centered around the test direction/Doppler point in the angle-Doppler domain (shown filled in Figure 1). Detection processing in the LPR is performed for this test direction/Doppler only. To test for targets in other angle-Doppler bins, a new LPR centered around the new look direction is formed. Interference suppression is achieved by adaptively combining data contained in the LPR. The covariance matrix corresponding to this LPR is estimated using secondary data from neighboring range cells. In Figure 1, the number of adaptive weights to be determined is 9, corresponding to 3 Doppler bins and 3 angle bins. The adaptive weights are given by

$$
w_{a}=R_{l}^{-1} \tilde{s}
$$

where, $R_{1}$ is the estimated angle-Doppler covariance matrix corresponding to the LPR of interest and $\widetilde{s}$ is the steering vector for the adaptive process. This vector should not be confused with either the spatial or temporal steering vectors. $\widetilde{S}$ is the space-time vector $\mathbf{S}=S_{s} \otimes S_{t}$ transformed to the angle-Doppler domain and is non-zero at only one point, i.e., for the case shown in Figure 1,

$$
\tilde{s}=\left[\begin{array}{llllllllll}
0 & 0 & 0 & 0 & 1 & 0 & 0 & 0 & 0 & 0
\end{array}\right]^{T}
$$

Adaptive weights are used to obtain a test statistic for hypothesis testing. In this paper, the modified sample matrix inversion (MSMI) statistic is used. The JDL-MSMI statistic is given by $\rho_{M S M I}=w_{a} \chi_{l} / w_{a} \widetilde{s}$ [Wang, 1995], where $\chi$ is the angle-Doppler data vector from the LPR at the range ring (cell) of interest.

\subsection{JDL using measured steering vectors}

The JDL algorithm, as developed above, assumes the receiver to be a linear array of equispaced, isotropic, point sensors. A real array, such as the MCARM array, may be planar. Further, an array of point sensors is not achievable in practice. Each array element must have non-zero physical size, which leads to mutual coupling between the elements. In a practical situation, therefore, the spatial steering vectors are not the Fourier coefficients. The spatial steering vector associated with a given angle is the amplitude and phase taper derived from 
measurements of a calibrated far field source. For a real array, the spatial steering vector in the preceding analysis must be replaced by the measured steering vector $\left(S_{s}^{m}\right)$, i.e., $\mathbf{S}=S_{s}^{m} \otimes S_{t}$.

The measured spatial steering vectors for different angle bins are not orthogonal to each other. Hence, the signal space-time steering vector is not localized to a single point in the angle-Doppler domain. Equation (4) must be modified to reflect the non-orthogonality of the steering vectors. For the JDL algorithm using 3 angle and 3 Doppler bins, the adaptive steering vector corresponding to angle bin (look direction) $n$ is given by

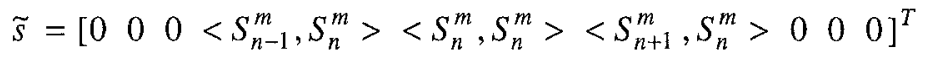

where, $\langle\bullet, \bullet\rangle$ denotes the inner product of two vectors, $S_{n}^{m}$ the measured spatial steering vector corresponding to angle bin $n$. The zeros in equation (6) are due to the orthogonal temporal steering vectors. Modifying the adaptive steering vector in this manner accounts for the non-orthogonality of the spatial steering vector. This formulation is easily extended to JDL algorithms utilizing more than 3 angle bins. Note that the measured steering vectors also transform the secondary data to angle-Doppler space. A DFT is valid for the transformation from time to Doppler space only.

A radar system transmits energy in some direction and the returns are processed for targets in that direction only. Hence, for each CPI, all Doppler bins are examined but the angle of interest remains constant. Therefore, for each CPI, the adaptive steering vector of equation (6) can be calculated a-priori.

\subsection{NUMERICAL EXAMPLES}

In this section, we present examples to illustrate the improvement in adaptive performance by considering the non-orthogonal nature of the spatial steering vectors. The examples use data from the Multi-Channel Airborne Radar Measurements (MCARM) database, a vast collection of clutter and signal measurements collected by an airborne radar over many flights with multiple acquisitions on each flight. The radar antenna is a 22 (2x11) rectangular array $(N=22)$. Included with the database is a set of measured steering vectors. These steering vectors are used in [Melvin, 1996] and also here for spatial processing of the data. The database includes clutter measurements and certain acquisitions include the signals (tones) of known Doppler shift and power from a Moving Target Simulator (MTS). The examples presented here are based on acquisitions from flight 5 . Each CPI comprises 128 pulses $(M=128)$.

Two examples are presented here to illustrate the improvement in detection performance. For each example, two scenarios are compared. In both scenarios, a DFT in the time domain and the measured steering vectors in space transform the space-time data to angle-Doppler space. The first scenario ignores the non-orthogonal nature of the spatial steering vectors [Melvin, 1996] and uses equation (5) as the adaptive steering vector. The second scenario accounts for the non-orthogonality and so uses equation (6) to evaluate the adaptive steering vector. In all examples 3 angle bins and 3 Doppler bins form the LPR. The covariance matrix of the interference is estimated using 2 guard cells and 36 secondary range cells centered about the range bin of interest.

\subsection{Example 1. Injected target}

In the first example, a synthetic target of fixed amplitude, direction, Doppler and range is injected into the MCARM data set. The amplitude and phase variation of the injected target across the 22 channels is obtained from the measured steering vectors. The amplitude of the injected target is chosen such that it remains undetected by nonadaptive digital beamforming/Doppler processing.

JDL processing is performed at the target angle bin, for a few range bins surrounding the injected target and for all Doppler bins. In this example, the figure of merit used to compare the two scenarios is the separation between the MSMI statistic at the target range/Doppler bin and the highest statistic at other range or Doppler bins (the largest false alarm statistic). A large separation implies a large difference between target and residual interference, i.e. improving the ability to detect the target.

In this example, the data from acquisition 575 on flight 5 is used. The parameters of the injected target are: Amplitude $=0.0003 \angle 0$, Angle bin $=0^{\circ}$ (Broadside), Doppler bin $=-9(-139.5 \mathrm{~Hz})$, Range bin $=290$ 
Figure 2 plots the MSMI statistic, as a function of Doppler bin for the two scenarios considered. In the first plot, the MSMI statistic at the target location is about $3.1 \mathrm{~dB}$ over the highest false alarm. However, when considering the non-orthogonal nature of the steering vectors, the separation between the target statistic and the nearest false alarm is $8.4 \mathrm{~dB}$. This is an improvement of $5.3 \mathrm{~dB}$ over the first case. Figure 3 plots the results of the same two cases as a function of range. In the first case, a strong false alarm $8.7 \mathrm{~dB}$ over the target is clearly visible. In the second case, the target is $2.5 \mathrm{~dB}$ over the nearest false alarm. Accounting for the non-orthogonal nature of the steering vectors yields an improvement of $11.2 \mathrm{~dB}$.

\subsection{MTS Tones:}

On flight 5, acquisition 152 includes clutter and tones from a moving target simulator (MTS) at pre-selected Doppler frequencies. Five tones are received at approximately $-800 \mathrm{~Hz}(0 \mathrm{~dB}),-600 \mathrm{~Hz}(-14 \mathrm{~dB}),-400 \mathrm{~Hz}(-20$ $\mathrm{dB}),-200 \mathrm{~Hz}(-26 \mathrm{~dB})$ and $0 \mathrm{~Hz}(-31 \mathrm{~dB})$. The data in this acquisition are returns from 128 pulses measured across 22 channels. The pulse repetition frequency for this flight was $1984 \mathrm{~Hz}$, hence the separation of $200 \mathrm{~Hz}$ corresponds to nearly 13 Dcppler bins. Using an acquisition with the MTS allows us to compare the performance of the JDL algorithm in the above scenarios using measured data. The MTS tones are processed like returns from moving targets. The presence of five MTS tones of differing amplitudes makes it difficult to define a unique figure of merit to compare the two scenarios. In this example, a visual inspection is used for comparison.

Figure 4 plots the MSMI statistic versus Doppler bin for the two cases considered. In the first plot, the five tones are clearly visible with the strongest tone at bin -53 spread over Doppler space. A few spurious tones are also visible. The second plot shows the results of the JDL algorithm modified by equation (6). The five tones are clearly visible and the spurious tones are completely suppressed.

\subsection{CONCLUSIONS}

This paper has shown that for a real antenna array, ignoring the non-orthogonal nature of the spatial steering vectors leads to significant degradation of the ability of the JDL algorithm to suppress interference. A simple modification to the adaptive steering vector can account for the non-orthogonality. This modification significantly improves on the performance of the traditional JDL algorithm. Examples using real data from the Multi-Channel Airborne Radar Measurement (MCARM) database illustrate the improvement in performance. This data set consists of real clutter, target measurements and MTS tones. In the case of the injected target and in the case of the MTS tones, the proposed modification results in significantly better performance over traditional joint domain localized processing.

\subsection{REFERENCES}

[Brennan, 1973] L.E. Brennan and I.S. Reed, "Theory of adaptive radar", IEEE Transactions on Aerospace and Electronic Systems, vol. 9, No. 3, pp. 237-252, March 1973.

[Melvin, 1996] W.L. Melvin and B. Himed "Comparative analysis of space-time adaptive algorithms with measured airborne data", In Proceedings of the $7^{\text {th }}$ International Conference on Signal Processing Applications \& Technology, Boston, MA, October 1996.

[Sloper, 1996] D. Sloper, et.al. "MCARM final report", Technical Report RL-TR-96-49, Rome Laboratory, April 1996.

[Wang, 1994] H. Wang and L. Cai, "On adaptive spatial-temporal processing for airborne surveillance radar systems", IEEE Transactions on Aerospace and Electronic Systems, vol. 30, No. 3, pp. 660-669, July 1994.

[Wang, 1995] H. Wang, "Space-time adaptive processing and its radar applications", Lecture Notes for ELE891, Syracuse University, Summer 1995. 
$3 \times 3 \mathrm{JDL}$. Ignoring non-orthogonality

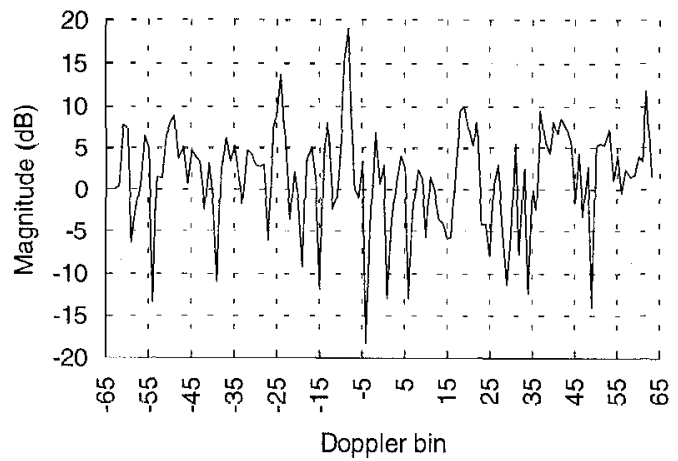

$3 \times 3$ Modified JDL.

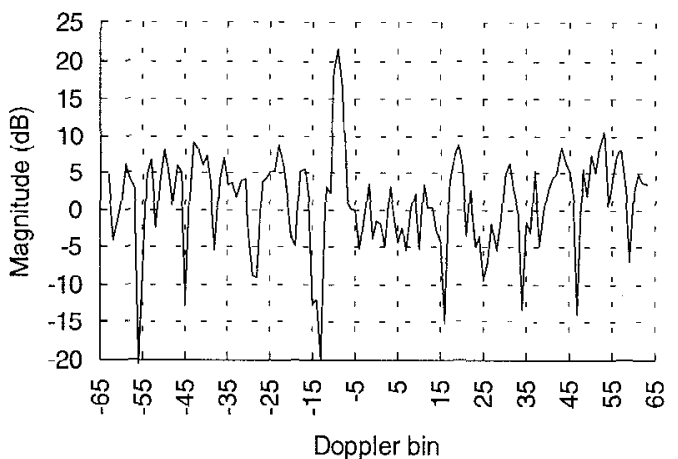

Figure 2. MSMI statistic v/s Doppler bin. Injected target

3x3 JDL. lgnoring non-orthogonality

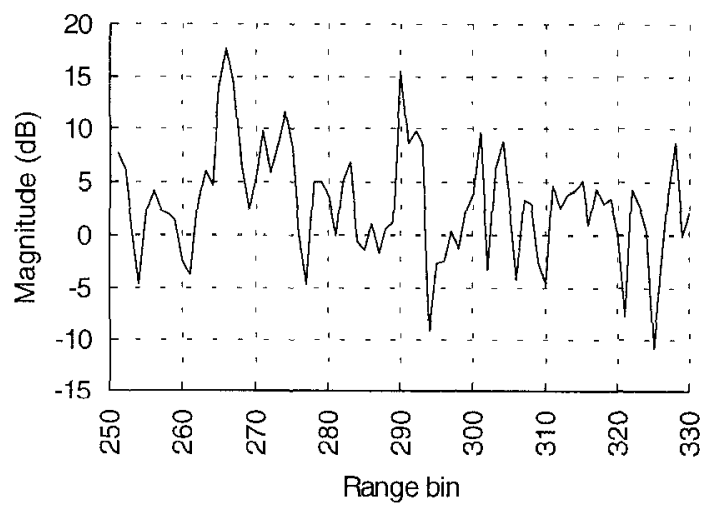

$3 \times 3$ Modified JDL

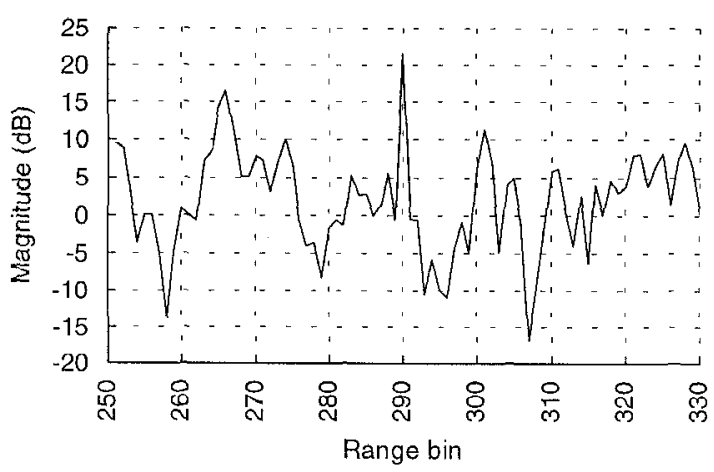

Figure 3. MSMI statistic v/s Range bin. Injected target

$3 \times 3$ JDL. lgnoring non-orthogonality

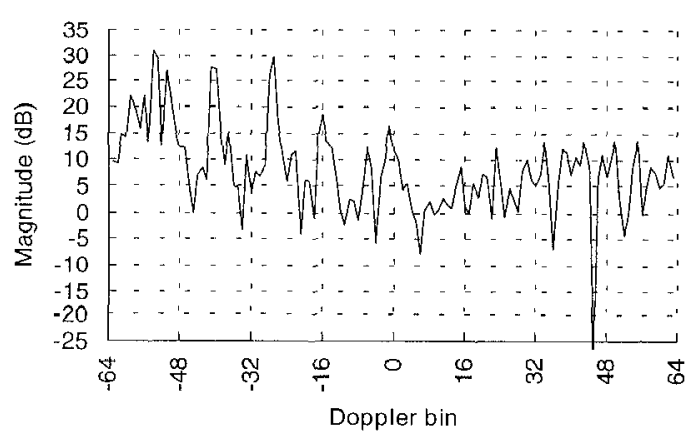

$3 \times 3$ JDL. Modified

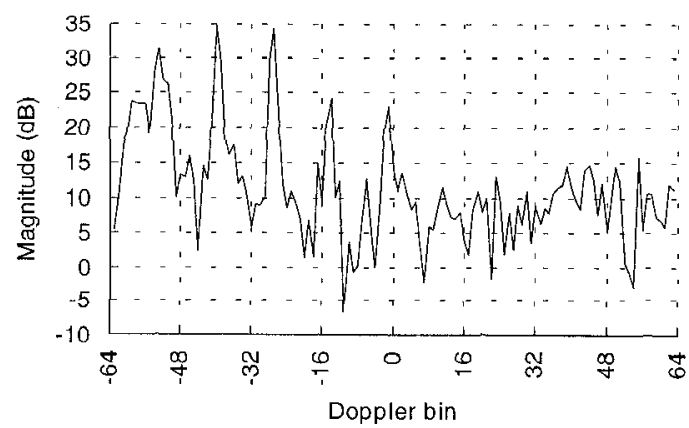

Figure 4. MSMI statistic v/s Doppler bin. MTS tones 